\title{
Standard exercise stress testing attenuates peripheral microvascular function in patients with suspected coronary microvascular dysfunction
}

\author{
Massimo Nardone ${ }^{1}$, Steven Miner ${ }^{1,2}$, Mary McCarthy ${ }^{2}$ and Heather Edgell ${ }^{1,2^{*}}$ (D)
}

\begin{abstract}
Background: The effect of exercise on the microvasculature of patients with suspected coronary microvascular dysfunction (CMD), assessed by reactive hyperemia peripheral arterial tonometry (RH-PAT; EndoPAT), is unknown. The present study aimed to determine if standard clinical exercise stress testing (GXT) affected peripheral microvascular function, as determined by the reactive hyperemia index (RHI and $L n R H I)$, in patients with suspected CMD.

Methods: In a cross-sectional study, patients $(n=76)$ were grouped based on whether the GXT was performed; 1 ) prior to (exercisers; $n=30$ ), or 2 ) after the vascular assessment (non-exercisers; $n=46$ ). Patients with an adenosine index of microvascular resistance $>25$, adenosine coronary flow reserve (CFR) $<2.0$, and/or acetylcholine CFR $<1.5$ were considered to have CMD $(n=42)$. RHI and LnRHI quantified finger pulse amplitude hyperemia following 5 min of forearm ischemia.

Results: LnRHI was lower in patients with CMD compared to patients without CMD, while LnRHI was also lower in exercisers compared to non-exercisers (LnRHI: CMD Non-Exercisers: $0.63 \pm 0.25$; CMD Exercisers: $0.54 \pm 0.19$; No CMD Non-Exercisers: $0.85 \pm 0.23$; No CMD Exercisers: $0.63 \pm 0.26$; Condition and Exercise Main Effects: Both $P<0.01$ ). In patients who did not exercise prior to the vascular assessment, the receiver operating characteristic curve (ROC) for LnRHI to predict CMD was 0.76 (95\% Cl: 0.62-0.91; $P<0.01)$. However, in patients who performed exercise prior to the vascular assessment, the ROC for $\mathrm{LnRHI}$ to predict CMD was 0.60 (95\% Cl: $0.40-0.81 ; P=0.34$ ).

Conclusions: $C M D$ is associated with impaired peripheral microvascular function and preceding acute exercise is associated with further reductions of LnRHI. Further, acute exercise abolished the capacity for RH-PAT to predict the presence of CMD in patients with chest pain and non-obstructive coronary arteries. RH-PAT measurements in patients with suspected CMD should not be conducted after exercise has been performed.
\end{abstract}

Keywords: Peripheral arterial tonometry, EndoPAT, Acute exercise, Augmentation index, Microvascular, Reactive hyperemia

\footnotetext{
* Correspondence: edgell@yorku.ca

${ }^{1}$ School of Kinesiology and Health Science, York University, 355 Norman Bethune College, Toronto, Ontario, Canada

${ }^{2}$ Southlake Regional Health Center, Newmarket, Ontario, Canada
}

C C The Author(s). 2021 Open Access This article is licensed under a Creative Commons Attribution 4.0 International License, which permits use, sharing, adaptation, distribution and reproduction in any medium or format, as long as you give appropriate credit to the original author(s) and the source, provide a link to the Creative Commons licence, and indicate if changes were made. The images or other third party material in this article are included in the article's Creative Commons licence, unless indicated otherwise in a credit line to the material. If material is not included in the article's Creative Commons licence and your intended use is not permitted by statutory regulation or exceeds the permitted use, you will need to obtain permission directly from the copyright holder. To view a copy of this licence, visit http://creativecommons.org/licenses/by/4.0/. The Creative Commons Public Domain Dedication waiver (http://creativecommons.org/publicdomain/zero/1.0/) applies to the data made available in this article, unless otherwise stated in a credit line to the data. 


\section{Background}

Reactive hyperemia peripheral arterial tonometry (RHPAT) is emerging as a novel measure of microvascular endothelial function, and is obtained by quantifying the hyperemic response within the finger microvasculature following brief ischemia [1, 2]. Peripheral microvascular function, using RH-PAT, predicts major adverse cardiovascular events in both health and disease [3-5]. In addition, peripheral endothelial dysfunction has been shown to relate to the function of coronary vasculature [6-8], implying that measuring peripheral endothelial function may be a time- and cost-efficient analysis to estimate coronary function in patients with suspected cardiovascular disease. Such analyses may be particularly relevant in patients with coronary microvascular dysfunction (CMD), a diseased characterized by abnormal coronary microvascular vasomotion and increased rates adverse cardiac outcomes [9-11]. In support of this, we have recently demonstrated that poor peripheral microvascular function, using RH-PAT, identifies patients with CMD [6], supporting its utility within clinical settings.

Despite the growing interest in RH-PAT assessments, factors known to influence RH-PAT remain poorly defined relative to other established measures of endothelial function, such as flow-mediated dilation [12, 13]. In particular, the impact of acute exercise on RH-PAT in patients with CMD is currently unknown. Studies quantifying endothelial function using flow-mediated dilation in healthy participants have highlighted that acute exercise briefly attenuates endothelial function, the magnitude of which is dependent on the duration, intensity, and type of exercise, as reviewed by Dawson et al. [14]. Unfortunately, these observations cannot necessarily be applied to RH-PAT, given that both flow-mediated dilation and RH-PAT are poorly correlated [15] and can be differentially influenced [15-17].

In addition, it is currently unclear whether patients with CMD experience an exaggerated reduction of endothelial function following exercise. Lower resting endothelial function is thought to lead to greater impairments of post-exercise vascular function [14], relevant considering that patients with $\mathrm{CMD}$ often present with reduced endothelial function $[6,18,19]$. Lastly, whether post-exercise alterations in endothelial function translate into diminished predictive capacity of RH-PAT for identifying patients with CMD remains unknown. Therefore, the present study aimed to: 1) confirm that patients with clinically diagnosed CMD exhibit impaired peripheral microvascular function, 2) determine if standard clinical exercise stress testing, commonly conducted within clinical practice, acutely affects peripheral microvascular function in patients with suspected $\mathrm{CMD}$, and 3) determine if exercise abolishes the predictive capacity of RH-PAT. We hypothesized that patients would have reduced peripheral microvascular function which would be further impaired by exercise such that RH-PAT measurements could no longer be used to predict the presence of CMD.

\section{Methods \\ Patient selection}

Seventy-six patients with suspected coronary microvascular disease (CMD) who attended the Cardiovascular Integrative Physiology Clinic at Southlake Regional Health Centre were recruited for this study. All nonexercisers included in this study also appear in Nardone et al. 2020 [6]. Subjects were included in this study if they underwent reactive hyperemia peripheral arterial tonometry (RH-PAT) assessments and coronary reactivity testing, to functionally assess peripheral and coronary microvascular function, respectively. Coronary reactivity testing was conducted if participants were experiencing typical or atypical chest pain and had angiographic evidence of normal coronary arteries (stenosis $<50 \%$ ). Detailed exclusion criteria for the current study have been described previously [6]. Briefly, these include: 1) hypertrophic cardiomyopathy, 2) coronary epicardial stenosis, 3) recent coronary artery intervention or bypass grafting, 4) heart failure $(\mathrm{EF}<40 \%)$, and 5) pulmonary hypertension induced by exercise. All procedures were approved by the Research Ethics Boards of Southlake Regional Health Centre and York University.

\section{Experimental protocol}

In a cross-sectional design, patients were grouped based on whether an exercise stress test was performed 1) prior to, or 2) following the vascular assessment. The exercise assessment consisted of a symptoms-limited maximal treadmill exercise stress test (GXT) using the Bruce protocol with concurrent 12-lead electrocardiography. The Duke Treadmill Score (DTS) was subsequently calculated, using the formula: DTS $=$ Exercise time $-(5 \mathrm{x}$ Max exercise ST) - (4 x Angina Index), as previously ascribed for risk classification [20]. Maximum exercise ST depression was the maximal depression present during exercise, where a maximal ST segment depression of $\geq 0.1 \mathrm{mV}$ was considered relevant myocardial ischemia. The angina index was ranked 0-2; 0 defined as no chest pain, 1 defined as non-limiting chest pain, and 2 defined as limiting chest pain. Heart rate and blood pressure were recorded both at rest and during maximal exercise. The time duration between the GXT and the vascular assessment was documented.

Prior to the vascular assessment, blood pressure was manually obtained, and height and body mass were recorded. Non-invasive assessment of peripheral microvascular function was conducted using reactive hyperemia peripheral arterial tonometry (RH-PAT) 
(EndoPat, Itamar Medical, Israel). Briefly, an applanation tonometry cuff was placed on the index finger of both hands. A blood pressure cuff was positioned on the right arm, immediately distal to the elbow joint. Five minutes of baseline recordings preceded $5 \mathrm{~min}$ of forearm ischemia, which involved supra-systolic cuff inflation to $\geq 200$ mmHg. Subsequently, the blood pressure cuff was quickly deflated to allow reperfusion of the distal limb for an additional $5 \mathrm{~min}$. The hyperemic response was quantified as the reactive hyperemic index (RHI) and the natural logarithm of RHI (LnRHI) (due to non-normal distribution of RHI) using automated algorithms of the EndoPat device [21]. More than 100 pulse amplitude waveforms of entire cardiac cycles were used during the baseline phase to determine augmentation index (AI), a measure of arterial stiffness. Further, due to the temporal relationship between heart rate and augmentation index [22], AI was also calculated at a controlled heart rate of 75 beats per minute (AI@75 bpm).

Approximately 5 months later, patients underwent coronary reactivity testing using the Doppler guidewire method, as previously described [6, 23]. In brief, a 0.014in pressure-temperature sensor-tipped Doppler guidewire was advanced into the left anterior descending coronary artery (LAD), which simultaneously measured proximal $(\mathrm{Pa})$ and distal $(\mathrm{Pd})$ coronary pressures, and the mean transit time of coronary flow (Tmn) via thermodilution [24]. The fractional flow reserve (FFR) was collected to confirm patients did not have significant coronary epicardial stenosis. An FFR $<0.80$ was considered hemodynamically significant coronary epicardial stenosis, and if present, patients were excluded. The Pa, $\mathrm{Pd}$, and Tmn were collected at rest and during intravenous adenosine $(140 \mu \mathrm{g} / \mathrm{kg} / \mathrm{min})$ and intracoronary acetylcholine $(20 \mu \mathrm{g}$ bolus $+100 \mu \mathrm{g}$ slow injection over $90 \mathrm{~s})$. During pharmacological hyperemia, the coronary flow reserve (CFR), and the index of microvascular resistance (IMR) were calculated as CFR $=\mathrm{Tmn}_{\text {baseline }} / \mathrm{Tmn}_{\text {hypere- }}$ mia , and $\mathrm{IMR}=\mathrm{Pd}_{\text {hyperemia }} \times \mathrm{Tmn}_{\text {hyperemia }}[25]$. As previously described by our group [6], an adenosine IMR $>$ 25 , adenosine $\mathrm{CFR}<2.0$, and/or acetylcholine CFR $<1.5$ was used as the diagnostic criteria for CMD.

\section{Data and statistical analysis}

Anthropometric data, GXT data, coronary reactivity testing data, and RH-PAT data were compared using a two by two factorial ANOVA. Levene's test of equality of variances was conducted prior to all ANOVA analyses. If a significant interaction was observed from the ANOVA, post hoc testing was conducted with the Bonferroni adjustment. The time between the GXT and the vascular assessment was compared between groups using an independent samples t-test. Medication regimen was grouped based on if subjects were taking; 1) any anti-hypertensive medications (i.e. one or more of the following: beta-adrenergic antagonist, angiotensin converting enzyme (ACE) inhibitor, angiotensin receptor blocker (ARB), diuretics, or a calcium channel blocker), and 2) any anti-cholesterol medications (i.e. one or more of the following: statin, or cholesterol absorption inhibitors). Medication usages were compared between groups using Chi squared testing. Further, use of either medication (i.e. anti-hypertensive and anti-cholesterol medications) and fasted state were utilized as covariates for ANCOVA analysis. To investigate the effect of time between the GXT and the vascular assessment, Pearson correlations between the LnRHI and the time between tests in exercisers with or without CMD were conducted. Lastly, to determine if exercise effects the capacity for RH-PAT to predict the presence of CMD, receiver operating characteristic (ROC) curve analysis was conducted to determine the ROC area under the curve $\left(\mathrm{ROC}_{\mathrm{AUC}}\right)$ in both exercisers and non-exercisers. All statistical analyses were performed using IBM SPSS Statistics 23 (Armonk, NY). Parametric data is presented as Mean $\pm \mathrm{SD}$, while non-parametric data is presented as Count (\%). Significance was defined as $P<0.05$.

\section{Results}

Between May 2017 and April 2019, 157 patients with suspected coronary microvascular dysfunction (CMD) attended the Cardiovascular Integrated Physiology Clinic at Southlake Regional Healthcare Centre for reactive hyperemia peripheral arterial tonometry (RH-PAT) assessments. After exclusion of patients who experienced technical errors during RH-PAT assessments $(n=9)$, patients who did not receive a referral for coronary reactivity testing $(n=25)$, patients who did not complete coronary reactivity testing $(n=33)$, patients who had hemodynamically significant coronary epicardial stenosis $(n=11)$, and patients who had technical difficulties during the coronary reactivity testing $(n=3)$, the sample consisted of 34 patients with normal coronary microvascular function and 42 patients with CMD (Fig. 1).

In patients with CMD, 25 patients did not exercise prior to assessment, and 17 patients exercised prior to assessment. In those without CMD, 21 patients did not exercise prior to assessment, and 13 patients exercised prior to assessment. There were no differences in anthropometrics, resting blood pressure, anti-cholesterol medications use, or fasted state between all groups $(P>$ 0.05 ; Table 1). Resting heart rate was elevated in exercisers compared to non-exercisers $(P=0.02$; Table 1$)$. Prior to coronary reactivity testing, a greater proportion of patients with confirmed CMD were taking antihypertensive medications compared to patients without CMD $(P<0.05$; Table 1$)$. Statistical analyses for the adenosine IMR, adenosine CFR, and acetylcholine CFR 


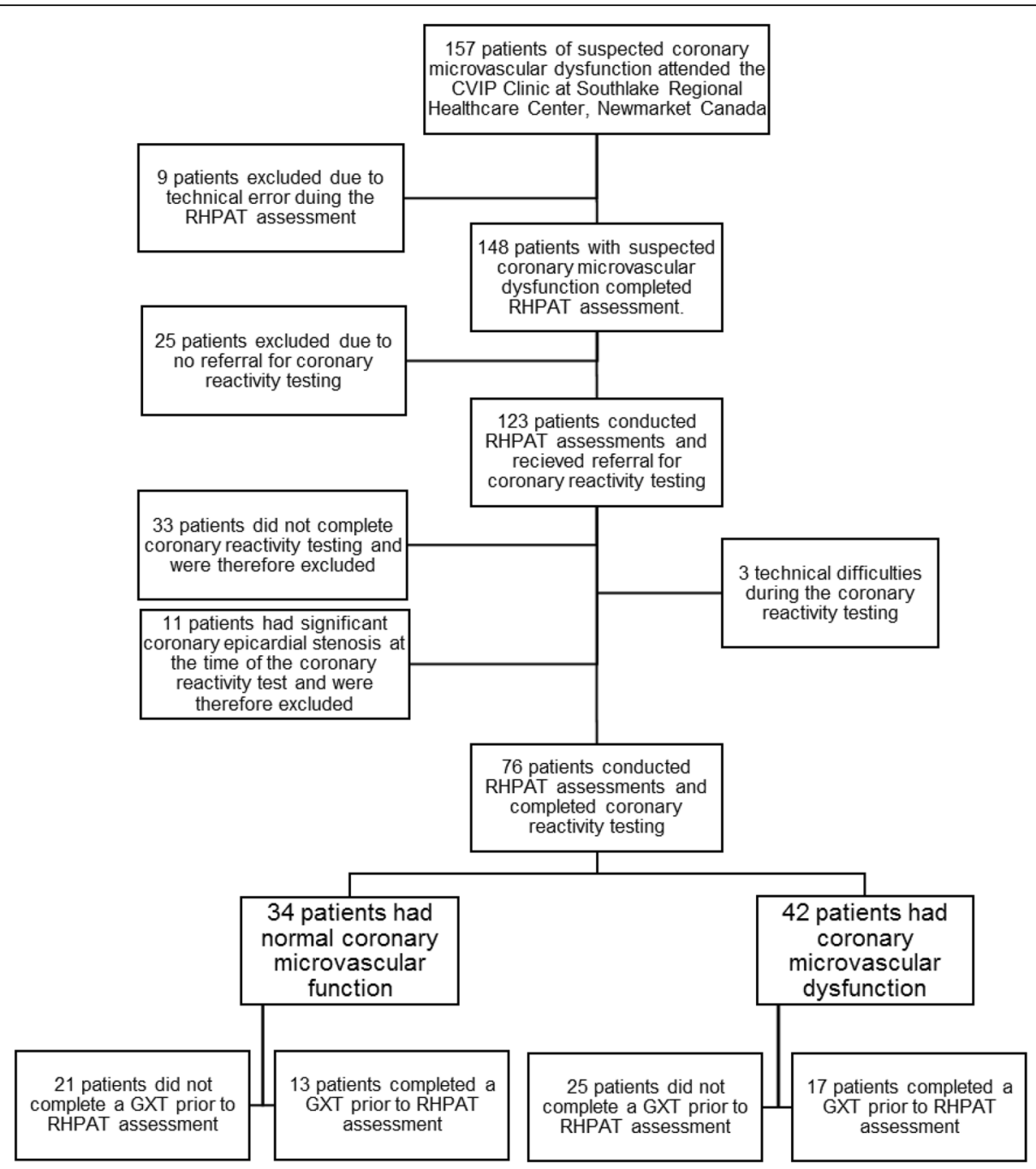

Fig. 1 Schematic of patients recruited from the Cardiovascular Integrative Physiology Clinic (CVIP) at Southlake Regional Health Centre. RH-PAT: Reactive Hyperemia Peripheral Arterial Tonometry; GXT Graded Exercise Test

were not conducted due to these variables being utilized as diagnostic criteria. However, the acetylcholine IMR was greater in patients with CMD compared to patients without $\mathrm{CMD}(\mathrm{P}<0.05$; Table 2$)$. Additionally, there was no difference in coronary microvascular function between non-exercisers and exercisers in both conditions $(P>0.05$; Table 2).

Five patients in both non-exerciser groups did not complete the graded exercise stress test at any time as a part of their normal clinical care (GXT; Table 3). In patients who were able to exercise, there were no differences in symptoms of exertional chest pain, exercise-induced myocardial ischemia, the Duke Treadmill Score between groups, or hemodynamics at peak exercise $(P>0.05$; Table 3$)$. Although there was an interaction between groups and exercise duration (Interaction: $P=0.048$; Table 3 ), post hoc testing indicated that there were no significant differences in exercise duration between groups $(P>0.05)$. In exercisers, the time between the GXT and the vascular assessment was not different between patients with and without CMD $(P>0.05$; Table 3$)$.

Prior to the adjustment for pharmaceutical use and fasting, the RHI and LnRHI were lower in patients with confirmed CMD compared to patients without CMD $(P<0.01)$, and exercisers demonstrated an attenuated RHI and LnRHI compared to non-exercisers $(P<0.01$; Table 4). Augmentation indices (AI and AI@75 bpm) were not different between patients with and without CMD $(P>0.05)$, and exercisers demonstrated an attenuated AI compared to non-exercisers $(P<0.01)$, while the AI@75 bpm were not different between exercisers and non-exercisers $(P>0.05$, Table 4). After adjusting for pharmaceutical use and fasting, the RHI and LnRHI were still lower in patients with confirmed CMD compared to patients without CMD 
Table 1 Anthropometrics, hemodynamics, medication use, and fasted state in patients with or without coronary microvascular dysfunction (CMD)

\begin{tabular}{|c|c|c|c|c|c|c|}
\hline & \multicolumn{3}{|l|}{ No CMD } & \multicolumn{3}{|l|}{ CMD } \\
\hline & All Patients & Non-EX & Exercisers & All Patients & Non-EX & Exercisers \\
\hline$n$ & 34 & 21 & 13 & 42 & 25 & 17 \\
\hline Sex, male, n (\%) & $13(38)$ & $7(33)$ & $6(46)$ & $13(31)$ & $8(32)$ & $5(29)$ \\
\hline Age (years) & $55 \pm 13$ & $58 \pm 13$ & $51 \pm 13$ & $60 \pm 13$ & $62 \pm 13$ & $57 \pm 11$ \\
\hline Body mass (kg) & $81.8 \pm 19.0$ & $76.8 \pm 18.4$ & $90.0 \pm 17.6$ & $78.1 \pm 15.4$ & $79.1 \pm 14.6$ & $76.7 \pm 16.8$ \\
\hline Height (m) & $1.69 \pm 0.10$ & $1.67 \pm 0.10$ & $1.73 \pm 0.08$ & $1.66 \pm 0.10$ & $1.66 \pm 0.08$ & $1.67 \pm 0.12$ \\
\hline $\mathrm{BMI}\left(\mathrm{kg} / \mathrm{m}^{2}\right)$ & $29 \pm 6$ & $27 \pm 6$ & $30 \pm 6$ & $28 \pm 4$ & $29 \pm 4$ & $27 \pm 5$ \\
\hline Resting SBP (mmHg) & $124 \pm 15$ & $124 \pm 18$ & $124 \pm 10$ & $123 \pm 12$ & $126 \pm 12$ & $117 \pm 11$ \\
\hline Resting DBP (mmHg) & $75 \pm 8$ & $76 \pm 8$ & $74 \pm 8$ & $76 \pm 9$ & $77 \pm 9$ & $74 \pm 9$ \\
\hline Resting HR (bpm) & $67 \pm 12$ & $62 \pm 9$ & $74 \pm 13^{\mathrm{a}}$ & $68 \pm 13$ & $65 \pm 13$ & $72 \pm 12^{a}$ \\
\hline \multicolumn{7}{|l|}{ Medication use, n (\%) } \\
\hline Anti-Hypertensive & $21(62)$ & $13(62)$ & $8(62)$ & $35(83) \dagger$ & $21(84)$ & $14(82)$ \\
\hline Anti-Cholesterol & $19(56)$ & $12(57)$ & $7(54)$ & $26(62)$ & $16(64)$ & $10(59)$ \\
\hline Fasted, n (\%) & $4(12)$ & $3(14)$ & $1(8)$ & $11(26)$ & $8(32)$ & $3(18)$ \\
\hline
\end{tabular}

Non-EX Non-Exercisers, CMD Coronary Microvascular Dysfunction, SBP Systolic Blood Pressure, DBP Diastolic Blood Pressure, HR Heart Rate, BMI Body Mass Index. ${ }^{a}$ indicates a significant exercise effect. + indicates a significant effect of CMD. Mean \pm SD

$(P<0.01$; Fig. 2). Further, RHI, LnRHI, and AI were still lower in exercisers compared to non-exercisers $(\mathrm{P}<0.01$; Fig. 2$)$, though there were no exercise or condition effects in the adjusted AI@75 bpm $(P>0.05$; Fig. 3). Correlations of the time between tests and the LnRHI were conducted for all exercisers combined, and a positive correlation was observed $(r=0.54, P<$ 0.01; Fig. 4). Further, in patients who did not exercise prior to the vascular assessment, the $\mathrm{ROC}_{\mathrm{AUC}}$ for LnRHI to predict CMD was 0.76 (95\% CI: $0.62-0.91$; $P<0.01)$. However, in patients who performed exercise prior to the vascular assessment, the $\mathrm{ROC}_{\mathrm{AUC}}$ for LnRHI to predict CMD was 0.60 (95\% CI: $0.40-0.81$; $P=0.34$ ). Lastly, covariate analysis of the adjusted model (data not shown) found that there were no effects of anti-hypertensive medication use, anticholesterol medication use, or fasted state on RHI, LnRHI, AI, or AI@75bpm $(P>0.05)$.

\section{Discussion}

The effect of acute exercise on peripheral microvascular function in patients with suspected coronary microvascular dysfunction (CMD) was previously unknown. First, in support of our recent findings [6], patients with CMD demonstrated impaired peripheral microvascular function. Second, consistent with studies in conduit arteries and healthy participants [14], acute exercise impaired microvascular function in patients with and without CMD. Third, contrary to our hypothesis, acute maximal exercise impaired peripheral microvascular function equally in patients with and without CMD. Fourth, acute graded exercise stress testing abolishes the capacity for RH-PAT to predict the presence of CMD in patients with chest pain and non-obstructive coronary arteries.

Most studies investigating vascular function following acute exercise have utilized brachial artery flow-

Table 2 Coronary flow and resistance responses to coronary infusions of adenosine and acetylcholine in patients with or without coronary microvascular dysfunction (CMD) undergoing coronary reactivity testing

\begin{tabular}{|c|c|c|c|c|c|c|}
\hline & \multicolumn{3}{|l|}{ No CMD } & \multicolumn{3}{|l|}{ CMD } \\
\hline & All Patients & Non-EX & Exercisers & All Patients & Non-EX & Exercisers \\
\hline \multicolumn{7}{|c|}{ Adenosine } \\
\hline IMR & $16.3 \pm 4.4$ & $15.8 \pm 4.6$ & $17.3 \pm 4.0$ & $26.2 \pm 13.3$ & $27.3 \pm 15.8$ & $24.6 \pm 8.5$ \\
\hline CFR & $4.0 \pm 1.6$ & $4.1 \pm 1.9$ & $3.7 \pm 1.1$ & $2.8 \pm 1.6$ & $2.7 \pm 1.9$ & $2.8 \pm 1.0$ \\
\hline \multicolumn{7}{|c|}{ Acetylcholine } \\
\hline IMR & $24.0 \pm 11.6$ & $24.4 \pm 11.9$ & $23.2 \pm 11.3$ & $39.7 \pm 21.7^{\mathrm{a}}$ & $40.1 \pm 19.6$ & $39.2 \pm 25.4$ \\
\hline CFR & $2.9 \pm 1.4$ & $2.7 \pm 1.6$ & $3.1 \pm 1.0$ & $1.9 \pm 1.3$ & $1.6 \pm 0.8$ & $2.3 \pm 1.7$ \\
\hline
\end{tabular}


Table 3 Symptoms-limited maximal exercise testing data in patients with or without coronary microvascular dysfunction (CMD)

\begin{tabular}{|c|c|c|c|c|}
\hline & \multicolumn{2}{|l|}{ No CMD } & \multicolumn{2}{|l|}{ CMD } \\
\hline & Non-EX & Exercisers & Non-EX & Exercisers \\
\hline$n$ & 18 & 13 & 20 & 17 \\
\hline LBBB, n (\%) & $1(6 \%)$ & $0(0)$ & $2(10)$ & $0(0)$ \\
\hline Time between tests (mins) & & $68 \pm 38$ & & $68 \pm 29$ \\
\hline Exercise duration (mins) & $8.36 \pm 3.11$ & $7.36 \pm 1.56$ & $7.26 \pm 3.24$ & $7.32 \pm 3.32$ \\
\hline \multicolumn{5}{|l|}{ Chest pain symptoms, n (\%) } \\
\hline Non-Limiting & $2(11)$ & $3(23)$ & $5(25)$ & $5(29)$ \\
\hline Limiting & $1(6)$ & $3(23)$ & $5(25)$ & $3(18)$ \\
\hline ST Depression, n (\%) & $10(56)$ & $8(62)$ & $11(55)$ & $5(29)$ \\
\hline Duke Treadmill Score (au) & $3 \pm 5$ & $1 \pm 5$ & $0 \pm 7$ & $3 \pm 5$ \\
\hline \multicolumn{5}{|l|}{ Hemodynamics } \\
\hline Peak HR (bpm) & $140 \pm 21$ & $137 \pm 23$ & $137 \pm 21$ & $136 \pm 18$ \\
\hline Peak SBP (mmHg) & $151 \pm 18$ & $154 \pm 18$ & $151 \pm 12$ & $138 \pm 20$ \\
\hline Peak DBP $(\mathrm{mmHg})$ & $78 \pm 11$ & $76 \pm 9$ & $80 \pm 10$ & $76 \pm 9$ \\
\hline
\end{tabular}

Non-EX Non-Exercisers, CMD Coronary Microvascular Dysfunction, DTS Duke Treadmill Score, LBBB left bundle branch block, HR heart rate, SBP systolic blood pressure, $D B P$ diastolic blood pressure. Time between tests represents the time between the exercise stress test and peripheral arterial tonometry in exercisers. No significant effect of exercise or CMD for all variables (All $P>0.05$ ). Mean \pm SD. Count (\%)

mediated dilation as a measure of conduit artery endothelial function. Using this method, post-exercise endothelial function has been reported inconsistently, as both increases [26-28] and decreases [27, 29-31] in brachial artery endothelial function have been observed. Inconsistent findings between studies could be due to differing intensity of exercise. Specifically, in young healthy participants, studies reporting decreased brachial artery endothelial function following exercise commonly utilize higher intensity exercise [31], while improved brachial artery endothelial function is commonly observed during low to moderate intensity exercise [26]. Additionally, the health/fitness status of participants may also influence the post-exercise vascular response. For instance, resistance exercise attenuated microvascular function in

Table 4 Unadjusted reactive hyperemia index $(\mathrm{RHI})$, natural logarithm of the RHI (LnRHI), augmentation index (AI), and augmentation index at 75 bpm (Al@75 bpm) in patients with or without coronary microvascular dysfunction (CMD). Patients were separated into groups who did and did not exercise prior to vascular assessment. Mean \pm SD

\begin{tabular}{|c|c|c|c|c|}
\hline & \multicolumn{2}{|l|}{ No CMD } & \multicolumn{2}{|l|}{ CMD } \\
\hline & Non-EX & Exercisers & Non-EX & Exercisers \\
\hline$\overline{R H I^{b}}$ & $2.41 \pm 0.55$ & $1.95 \pm 0.56^{\mathrm{a}}$ & $1.93 \pm 0.53$ & $1.74 \pm 0.35^{\mathrm{a}}$ \\
\hline $\operatorname{LnRHI}^{b}$ & $0.85 \pm 0.23$ & $0.63 \pm 0.26^{a}$ & $0.63 \pm 0.25$ & $0.54 \pm 0.19^{a}$ \\
\hline $\mathrm{Al}$ & $25.2 \pm 19.1$ & $8.5 \pm 17.8^{a}$ & $23.7 \pm 25.3$ & $13.7 \pm 16.4^{a}$ \\
\hline Al@75 bpm & $16.9 \pm 16.6$ & $8.1 \pm 13.6$ & $17.4 \pm 25.8$ & $12.0 \pm 14.0$ \\
\hline
\end{tabular}

Non-EX Non-Exercisers, CMD Coronary Microvascular Dysfunction, $R H I$ Reactive Hyperemia Index, LnRHI Natural logarithm of the RHI, Al Augmentation Index, Al@75 bpm Augmentation Index at $75 \mathrm{bpm} .{ }^{\mathrm{a}}$ indicates a significant exercise effect. ${ }^{b}$ indicates a significant effect of CMD. Mean \pm SD sedentary participants, though this was not observed in trained participants [32]. Similar findings in overweight participants have been observed, such that active participants increased, and inactive participants decreased, brachial artery endothelial function following acute exercise [27]. Patients with intermittent claudication also reported attenuated brachial artery endothelial function following higher intensity treadmill exercise [29]. In the current study, suspected CMD patients who conducted symptom-limited maximal treadmill exercise prior to RH-PAT testing exhibited an attenuation of RHI and LnRHI, supporting previous work that both higher intensity exercise, and patients with cardiovascular disease demonstrate attenuated peripheral endothelial function.

The mechanisms responsible for reducing microvascular function in the current study are unclear. Previous investigators have proposed that attenuated endothelial function due to the performance of exercise may be a result of elevated resting conduit artery diameter and blood flow [14]. This observation is particularly relevant considering that brachial artery diameter has been shown to influence the RH-PAT response, such that a larger brachial artery diameter is associated with a higher baseline pulse amplitude and a lower RHI [33]. Therefore, these post-exercise vascular alterations may act to diminish the vasodilatory reservoir and mathematically reduce the RH-PAT response. Alternatively, oxidative stress is increased during high intensity exercise [34, 35], facilitating a reduction in endothelial function following acute exercise in health [27, 36] and disease [29]. Since oxidative stress has been shown to be elevated in patients with CMD [37], we had hypothesized that 
A

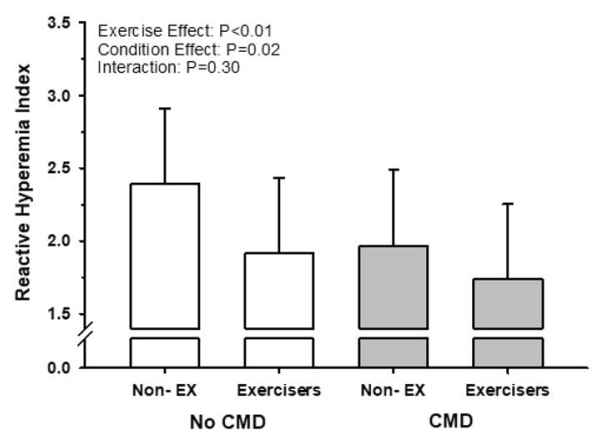

B

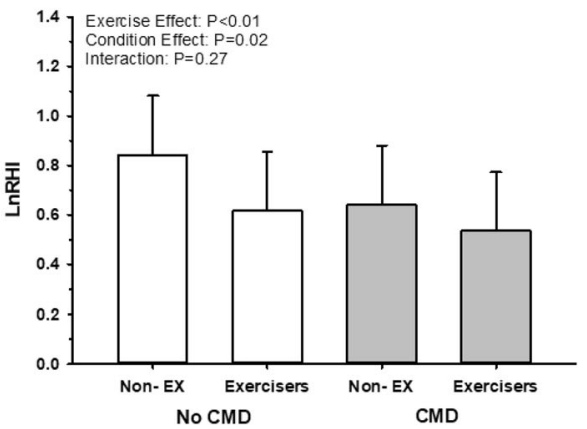

Fig. 2 Adjusted reactive hyperemia index $(\mathrm{RHI} ; \mathbf{a})$ and natural logarithm of the $\mathrm{RHI}(\mathrm{LnRHI}$; b) in patients with or without coronary microvascular dysfunction (CMD). Patients were separated into groups who did and did not exercise prior to vascular assessment. White bars represent patients without CMD. Grey bars represent patients with CMD. Non-EX indicates patients who did not exercise prior to assessment. Mean \pm SD

baseline impairments in peripheral endothelial function in CMD patients would exacerbate post-exercise microvascular function compared to the non-CMD group, yet this was not observed. We suggest that measurements of oxidative stress should be added to future longitudinal investigations of the effect of exercise in CMD. Lastly, RH-PAT is strongly associated with resting sympathetic activity [38], to a greater extent than brachial artery flow mediated dilation [39]. Given that sympathetic activity is elevated following exercise, the reduced RHI and LnRHI in the current study may potentially be related to increased sympathetic activity, rather than reduced endothelial function caused by oxidative stress.

Contrary to previous reports $[40,41]$, the present study observed that measures of arterial stiffness were not different in patients with and without CMD. Several studies observed that both the aortic augmentation index [40] and the carotid artery augmentation index [41] were associated with the CFR response to intravenous adenosine in patients with CMD, implying that arterial stiffness in the large, mainly elastic arteries, is elevated in patients with CMD. In the current study, augmentation index was obtained from the finger microvasculature using the EndoPAT device, suggesting that arterial stiffness in the peripheral vasculature is similar in both conditions. We did not observe a significant effect of exercise on augmentation index normalized to heart rate (AI@75 bpm) indicating that acute exercise did not influence peripheral arterial stiffness in patients with suspected CMD. The validity of the augmentation indices obtained from the EndoPAT device have yet to be compared to a gold-standard measurement such as applanation tonometry of conduit vessels.

\section{Clinical relevance}

The use of RH-PAT is a clinically appealing method of measuring peripheral microvascular function, given that RH-PAT assessments are relatively cost efficient,
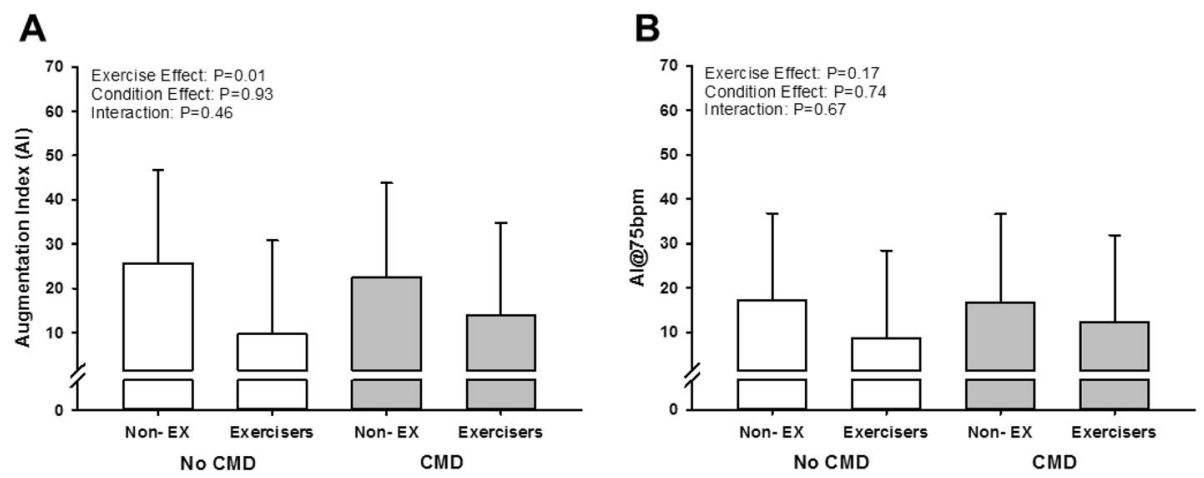

Fig. 3 Adjusted augmentation index (Al; a) and augmentation index at 75 bpm (Al@75 bpm; b) in patients with or without coronary microvascular dysfunction (CMD). Patients were separated into groups who did and did not exercise prior to vascular assessment. White bars represent patients without CMD. Grey bars represent patients with CMD. Non-EX indicates patients who did not exercise prior to assessment. Mean \pm SD 


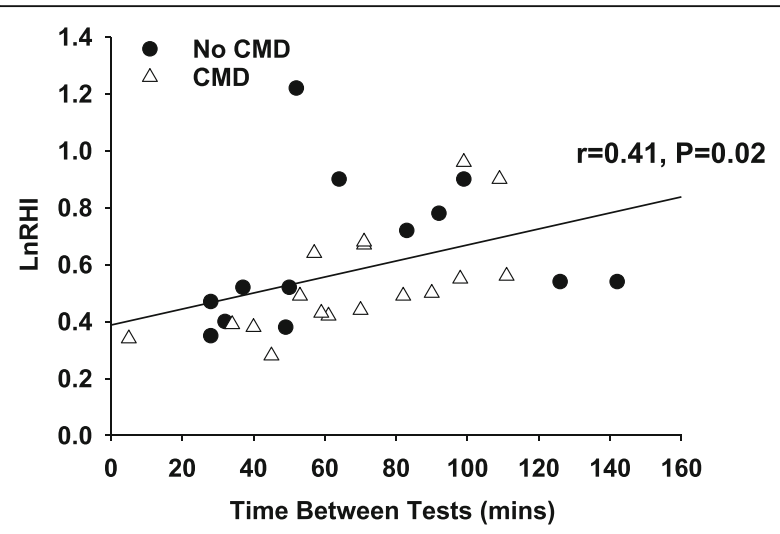

Fig. 4 Correlation between the natural logarithm of the reactive hyperemia index $(\mathrm{LnRHI})$ and the time between the graded exercise stress test and the vascular assessment in patients who exercised prior to the vascular assessment. Black circles represent patients without coronary microvascular dysfunction (CMD). White triangles represent patients with $\mathrm{CMD}$

operator independent, possess objective and automated analyses [2], and are highly reproducible [42, 43]. However, despite growing interest, the standardizations for RH-PAT assessments have not been determined in health or disease relative to other established measures of vascular function, such as flow-mediated dilation [12, 13]. Further, flowmediated dilation and RH-PAT are poorly correlated [15] and can be influenced differently by aging [15], exercise training [44], and medication use [16], suggesting that established variables known to alter flowmediated dilation do not necessarily alter RH-PAT. These observations further exemplify the importance of establishing RH-PAT specific standardizations. Our data suggest that RHI and LnRHI are reduced by standard exercise stress testing, abolishing the predictive capacity of RH-PAT in identifying patients with CMD. Notably, the deterioration of RH-PAT due to CMD and the deterioration due to exercise in patients without CMD were similar. Therefore, caution should be used in interpreting RH-PAT results when completed after exercise stress testing.

Additionally, our data show that RHI and LnRHI were not influenced by pharmacological use or fasting. In particular, the observation that fasting did not demonstrate a significant influence is important for CMD patients since fasting prior to exercise could lead to hypoglycemia during their clinical visit and/or exercise stress test, given the high prevalence of diabetes mellitus $[45,46]$.

\section{Limitations}

We acknowledge several important considerations. First, this study was a cross-sectional study design. To fully elucidate the relationship between RHI and acute exercise, a repeated measures study design should be completed. To date, no study has investigated the relationship between $\mathrm{RHI}$ and exercise in patients with suspected CMD, and these results provide strong evidence to continue this work. Second, since the time delay between the RH-PAT assessment and the diagnostic coronary reactivity testing was $\sim 5$ months, we cannot rule out that peripheral vascular function could have been altered within this time period due to changes in lifestyle or severity of condition, despite medication use being unchanged. However, the relationship between peripheral vascular function and the presence of CMD would likely have been lessened due to the time delay, decreasing the likelihood of finding a significant difference. Thirdly, although patients with and without CMD had similar exercise tolerances during the GXT (suggesting equal levels of cardiorespiratory fitness), this study did not objectively assess physical activity or oxygen consumption via a full cardiopulmonary exercise test. Lastly, lower physical activity has been linked with impaired endothelial function following acute exercise [30] potentially influencing our results. Therefore, future studies should obtain accurate measurements of fitness using accelerometry and oxygen consumption.

\section{Conclusions}

These data support previous findings that patients with confirmed CMD have impaired peripheral microvascular function compared to patients without CMD and are the first data to suggest that exercise stress testing impairs peripheral microvascular function in all patients with suspected CMD. Further, in exercisers with and without $\mathrm{CMD}$, there was a positive relationship between peripheral microvascular function and the time between exercise testing and vascular assessment. We recommend that RH-PAT measurements should not be conducted after exercise has been performed.

\section{Abbreviations \\ Al: Augmentation index; Al@75 bpm: Augmentation index at 75 bpm; CFR: Coronary flow reserve, $C$ CMD: Coronary microvascular dysfunction; DBP: Diastolic blood pressure; FFR: Fractional flow reserve; GXT: Graded exercise stress test; HR: Heart rate; IMR: Index of microvascular resistance; LBBB: Left bundle branch block; LnRHI: Natural logarithm of the reactive hyperemia index; Pa: Pressure at proximal coronary segment; Pd: Pressure at distal coronary segment; RH-PAT: Reactive hyperemia peripheral arterial tonometry; RHI: Reactive hyperemia index; SBP: Systolic blood pressure; Tmn: Mean transit time}

\section{Acknowledgements}

We would like to acknowledge that the work presented in this manuscript is part of Massimo Nardone's M.Sc. Thesis: Comparison of non-invasive peripheral vascular function to invasive measures of coronary function in patients with suspected coronary microvascular dysfunction.

Authors' contributions

MN, SM, MM, \& HE performed experiments; MN, SM, MM, \& HE analyzed data; MN \& HE interpreted the results; MN \& HE drafted manuscript. All 
authors provided feedback and revisions and approved the final manuscript version prior to submission.

\section{Funding}

This research was supported by the Natural Sciences and Engineering Research Council of Canada (NSERC), Canadian Foundation for Innovation $(\mathrm{CFI})$, Ontario Research Fund (ORF), and St Jude Medical. This funding was responsible for the infrastructure used for data collection and analysis. M.N. was supported by a CIHR Fredrick Banting and Charles Best Canada Graduate Scholarship and the Heart of Gold cardiac research fund. This funding was responsible for funding M.N. for data collection, analysis, interpretation of data, and writing the manuscript.

\section{Availability of data and materials}

The datasets used and analyzed during the current study are available from the principal researcher (HE: edgell@yorku.ca) upon reasonable request.

\section{Ethics approval and consent to participate}

The participants gave their informed consent in written form at their first visit to the research center and the trial was conducted according to the Declaration of Helsinki. The study was approved by the Research Ethics Committee at Southlake Regional Health Centre and York University.

\section{Consent for publication}

Not applicable.

\section{Competing interests}

The authors declare no conflicts of interest relevant to the content of this study.

Received: 26 May 2020 Accepted: 17 February 2021 Published online: 27 February 2021

\section{References}

1. Celermajer DS. Reliable endothelial function testing. Circulation. 2008;117: 2428-30.

2. Hamburg NM, Benjamin EJ. Assessment of endothelial function using digital pulse amplitude tonometry. Trends Cardiovasc Med. 2009;19:6-11.

3. Kuznetsova T, Vlierberghe EV, Knez J, Szczesny G, Thijs L, Jozeau D, et al. Association of digital vascular function with cardiovascular risk factors: a population study. BMJ Open. 2014;4:e004399.

4. Matsue Y, Yoshida K, Nagahori W, Ohno M, Suzuki M, Matsumura A, et al. Peripheral microvascular dysfunction predicts residual risk in coronary artery disease patients on statin therapy. Atherosclerosis. 2014;232:186-90.

5. Matsuzawa Y, Sugiyama S, Sumida H, Sugamura K, Nozaki T, Ohba K, et al. Peripheral endothelial function and cardiovascular events in high-risk patients. J Am Heart Assoc. 2013;2:e000426.

6. Nardone $\mathrm{M}$, Miner S, McCarthy $\mathrm{M}$, Ardern $\mathrm{Cl}$, Edgell $\mathrm{H}$. Noninvasive microvascular indices reveal peripheral vascular abnormalities in patients with suspected coronary microvascular dysfunction. Can J Cardiol. 2020;36: 1289-97.

7. Park C-S, Youn H-J, Kim J-H, Cho E-J, Jung H-O, Jeon H-K, et al. Relation between peripheral vascular endothelial function and coronary flow reserve in patients with chest pain and normal coronary angiogram. Int J Cardiol. 2006:113:118-20.

8. Sax FL, Cannon ROI, Hanson C, Epstein SE. Impaired forearm vasodilator reserve in patients with microvascular angina. N Engl J Med. 1987;317: 1366-70.

9. Camici PG, Crea F. Coronary microvascular dysfunction. N Engl J Med. 2007; 356:830-40

10. Bairey Merz CN, Pepine CJ, Walsh MN, Fleg JL. Ischemia and no obstructive coronary artery disease (INOCA): developing evidence-based therapies and research agenda for the next decade. Circulation. 2017;135:1075-92.

11. Pepine CJ, Anderson RD, Sharaf BL, Reis SE, Smith KM, Handberg EM, et al. Coronary microvascular reactivity to adenosine predicts adverse outcome in women evaluated for suspected ischemia: results from the National Heart, lung and blood institute WISE (Women's ischemia syndrome evaluation) study. J Am Coll Cardiol. 2010:55:2825-32.

12. Thijssen DHJ, Black MA, Pyke KE, Padilla J, Atkinson G, Harris RA, et al. Assessment of flow-mediated dilation in humans: a methodological and physiological guideline. Am J Physiol - Heart Circ Physiol. 2011;300:H2-12.
13. Thijssen DHJ, Bruno RM, van Mil ACCM, Holder SM, Faita F, Greyling A, et al. Expert consensus and evidence-based recommendations for the assessment of flow-mediated dilation in humans. Eur Heart J. 2019;40:2534-47.

14. Dawson EA, Green DJ, Cable NT, Thijssen DHJ. Effects of acute exercise on flow-mediated dilatation in healthy humans. J Appl Physiol. 2013;115:1589-98.

15. Hamburg NM, Palmisano J, Larson MG, Sullivan LM, Lehman BT, Vasan RS, et al. Relation of brachial and digital measures of vascular function in the community: the Framingham heart study. Hypertension. 2011;57:390-6.

16. Kabaklić A, Fras Z. Moderate-dose atorvastatin improves arterial endothelial function in patients with angina pectoris and normal coronary angiogram: a pilot study. Arch Med Sci AMS. 2017;13:827-36.

17. Babcock MC, DuBose LE, Witten TL, Brubaker A, Stauffer BL, Hildreth KL, Moreau KL. Assessment of macrovascular and microvascular function in aging males. J Appl Physiol (1985). 2021;130(1):96-103.

18. Lekakis JP, Papamichael CM, Vemmos CN, Voutsas AA, Stamatelopoulos SF, Moulopoulos SD. Peripheral vascular endothelial dysfunction in patients with angina pectoris and Normal coronary arteriograms. J Am Coll Cardiol. 1998;31:541-6.

19. Masci PG, Laclaustra M, Lara JG, Kaski JC. Brachial artery flow-mediated dilation and myocardial perfusion in patients with cardiac syndrome X. Am J Cardiol. 2005;95:1478-80.

20. Mark DB, Shaw L, Harrell FE, Hlatky MA, Lee KL, Bengtson JR, et al. Prognostic value of a treadmill exercise score in outpatients with suspected coronary artery disease. N Engl J Med. 1991;325:849-53.

21. Kuvin JT, Patel AR, Sliney KA, Pandian NG, Sheffy J, Schnall RP, et al. Assessment of peripheral vascular endothelial function with finger arterial pulse wave amplitude. Am Heart J. 2003;146:168-74.

22. Wilkinson IB, MacCallum H, Flint L, Cockcroft JR, Newby DE, Webb DJ. The influence of heart rate on augmentation index and central arterial pressure in humans. J Physiol. 2000;525(Pt 1):263-70

23. Nardone M, McCarthy M, Ardern Cl, Edgell H, Toleva O, Nield LE, et al. Characterization of the human coronary microvascular response to multiple hyperemic agents. CJC Open. 2021;3(2):133-41.

24. Pijls NHJ, Bruyne BD, Smith L, Aarnoudse W, Barbato E, Bartunek J, et al. Coronary thermodilution to assess flow reserve: validation in humans. Circulation. 2002;105:2482-6.

25. Kobayashi Y, Fearon WF, Honda Y, Tanaka S, Pargaonkar V, Fitzgerald PJ, et al. Effect of sex differences on invasive measures of coronary microvascular dysfunction in patients with angina in the absence of obstructive coronary artery disease. JACC Cardiovasc Interv. 2015;8:1433-41.

26. Padilla J, Harris RA, Fly AD, Rink LD, Wallace JP. The effect of acute exercise on endothelial function following a high-fat meal. Eur J Appl Physiol. 2006; 98:256-62.

27. Harris RA, Padilla J, Hanlon KP, Rink LD, Wallace JP. The flow-mediated dilation response to acute exercise in overweight active and inactive men. Obesity. 2008;16:578-84.

28. Tinken TM, Thijssen DHJ, Hopkins N, Black MA, Dawson EA, Minson CT, et al. Impact of shear rate modulation on vascular function in humans. Hypertension. 2009;54:278-85.

29. Silvestro A, Scopacasa F, Oliva G, de Cristofaro T, luliano L, Brevetti G. Vitamin $C$ prevents endothelial dysfunction induced by acute exercise in patients with intermittent claudication. Atherosclerosis. 2002;165:277-83.

30. Hwang I-C, Kim K-H, Choi W-S, Kim H-J, Im M-S, Kim Y-J, et al. Impact of acute exercise on brachial artery flow-mediated dilatation in young healthy people. Cardiovasc Ultrasound. 2012;10:39.

31. Birk GK, Dawson EA, Batterham AM, Atkinson G, Cable T, Thijssen DHJ, et al. Effects of exercise intensity on flow mediated dilation in healthy humans. Int J Sports Med. 2013;34:409-14.

32. Durand MJ, Kodlipet D, Bian J-T, Das E, Vidovich M, Gutterman DD, et al Acute exertion elicits a H2O2-dependent vasodilator mechanism in the microvasculature of exercise-trained but not sedentary adults. Hypertension. 2015;65:140-5

33. Heffernan KS, Karas RH, Mooney PJ, Patel AR, Kuvin JT. Pulse wave amplitude is associated with brachial artery diameter: implications for gender differences in microvascular function. Vasc Med. 2010;15:39-45.

34. Finaud J, Lac G, Filaire E. Oxidative stress: relationship with exercise and training. Sports Med. 2006:36:327-58

35. Ji LL. Exercise-induced modulation of antioxidant defense. Ann N Y Acad Sci. 2002:959:82-92.

36. Johnson BD, Padilla J, Wallace JP. The exercise dose affects oxidative stress and brachial artery flow-mediated dilation in trained men. Eur J Appl Physiol. 2012;112:33-42. 
37. Sakr SA, Abbas TM, Amer MZ, Dawood EM, El-Shahat N, Aal IAA, et al. Microvascular angina. The possible role of inflammation, uric acid, and endothelial dysfunction. Int Heart J. 2009;50:407-19.

38. Sverrisdóttir YB, Jansson LM, Hägg U, Gan L-M. Muscle sympathetic nerve activity is related to a surrogate marker of endothelial function in healthy individuals. PLoS One. 2010;5:e9257.

39. Tomiyama H, Yoshida M, Higashi Y, Takase B, Furumoto T, Kario K, et al. Autonomic nervous activation triggered during induction of reactive hyperemia exerts a greater influence on the measured reactive hyperemia index by peripheral arterial tonometry than on flow-mediated vasodilatation of the brachial artery in patients with hypertension. Hypertens Res. 2014;37: 914-8.

40. Nichols WW, Denardo SJ, Davidson JB, Huo T, Bairey Merz CN, Pepine CJ. Association of aortic stiffness and wave reflections with coronary flow reserve in women without obstructive coronary artery disease: an ancillary study from the National Heart, Lung, and Blood Institutesponsored Women's ischemia syndrome evaluation (WISE). Am Heart J. 2015;170:1243-54

41. Saito M, Okayama H, Nishimura K, Ogimoto A, Ohtsuka T, Inoue K, et al. Possible link between large artery stiffness and coronary flow velocity reserve. Heart. 2008;94:e20.

42. Brant LCC, Barreto SM, Passos VMA, Ribeiro ALP. Reproducibility of peripheral arterial tonometry for the assessment of endothelial function in adults. J Hypertens. 2013;31:1984-90.

43. Liu J, Wang J, Jin Y, Roethig HJ, Unverdorben M. Variability of peripheral arterial tonometry in the measurement of endothelial function in healthy men. Clin Cardiol. 2009:32:700-4.

44. Cornelissen VA, Onkelinx S, Goetschalckx K, Thomaes T, Janssens S, Fagard $\mathrm{R}$, et al. Exercise-based cardiac rehabilitation improves endothelial function assessed by flow-mediated dilation but not by pulse amplitude tonometry. Eur J Prev Cardiol. 2014;21:39-48.

45. Di Carli MF, Charytan D, McMahon GT, Ganz P, Dorbala S, Schelbert HR. Coronary circulatory function in patients with the metabolic syndrome. Nucl Med. 2011;52:1369-77.

46. Nahser PJ, Brown RE, Oskarsson H, Winniford MD, Rossen JD. Maxima coronary flow reserve and metabolic coronary vasodilation in patients with diabetes mellitus. Circulation. 1995;91:635-40.

\section{Publisher's Note}

Springer Nature remains neutral with regard to jurisdictional claims in published maps and institutional affiliations.

Ready to submit your research? Choose BMC and benefit from:

- fast, convenient online submission

- thorough peer review by experienced researchers in your field

- rapid publication on acceptance

- support for research data, including large and complex data types

- gold Open Access which fosters wider collaboration and increased citations

- maximum visibility for your research: over $100 \mathrm{M}$ website views per year

At BMC, research is always in progress.

Learn more biomedcentral.com/submissions 